\title{
La búsqueda de información con fines académicos entre el alumnado universitario
}

\author{
Rubén Comas*, Jaume Sureda*, Miquel Pastor ${ }^{\star \star}$, Mercé Morey*
}

Resumen: En este trabajo se presentan los resultados de un estudio sobre la autovaloración de los conocimientos y habilidades del alumnado pregraduado de la Universidad de las Islas Baleares (UIB) para la búsqueda de información con fines académicos. También se describen diversos aspectos sobre la utilización de algunos servicios bibliotecarios así como de Internet. El estudio se basó en la aplicación de un cuestionario a un total de 1.025 universitarios seleccionados aleatoriamente. De los resultados alcanzados se desprende que el alumnado universitario se considera, por norma general, competente en el manejo de Internet para documentarse con fines académicos, pero no en el uso de las bibliotecas. Lógicamente, la frecuencia de uso de Internet también es muy superior a la de los otros recursos de búsqueda de información académica. Cabe plantear, en este sentido, la necesidad de mejorar las competencias y estrategias de documentación del alumnado basadas en el uso de las bibliotecas, recurso que no excluye el empleo de Internet, pero que a buen seguro puede mejorar la búsqueda documental en aspectos como el rigor, la fiabilidad y la potencialidad de cubrir mucho mejor las necesidades informacionales del alumnado.

Palabras clave: competencias académicas, alfabetización informacional, estrategias de búsqueda documental.

\section{Strategies used by university students in searching for information for academic purposes}

Abstract: This paper presents the results of a study of undergraduate students at the University of the Balearic Islands (UIB) focused on 1) a self-assessment of their knowledge and skills for using library services and the Internet in searching for information for academic purposes; 2) the frequency of their use of library services in general, and of the UIB's library catalogue and the Internet for academic purposes, in particular. The study is based on a questionnaire sent to 1,025 randomly selected university students. The results obtained suggest that university students consider themselves, in general terms, competent in using the Internet for searching information for academic purposes, but not in using libraries for the same purpose. Logically, the frequency of Internet usage is also far superior to that of other resources used to search for academic information.

* Departamento de Pedagogía Aplicada y Psicología de la Educación de la Universidad de las Islas Baleares. Correo-e: rubencomas@uib.es; sureda.negre@gmail.com; merce.morey@uib.es

** Servicio de Bibliotecas y Documentación de la Universidad de las Islas Baleares. Correo-e: miquel.pastor@uib.cat.

Recibido: 28-01-2010; 2. ${ }^{a}$ versión: 25-03-2010; aceptado: 28-03-2010. 
Therefore, we believe it is necessary to improve the documentation skills and strategies for students' use of libraries. This should not exclude the use of the Internet, but rather complement it for enhancing search results in regards to rigor, reliability and the potential to cover informational needs of an academic nature.

Keywords: Academic competences, information literacy, information search strategies.

\section{Introducción}

Los desarrollos de las Tecnologías de la Información y la Comunicación (TIC) no sólo han generado nuevos contextos, estilos relacionales y códigos de acción en los escenarios de enseñanza-aprendizaje, sino que también han propiciado la aparición de nuevos espacios, componentes y criterios de búsqueda, localización y gestión de la información de carácter académico. Estos cambios han generado el surgimiento de nuevas necesidades que ponen en relación el potencial de los recursos con la manera en que los usuarios los empleamos y aprovechamos; estas necesidades se han conceptualizado en la literatura reciente con el término "alfabetizaciones múltiples" —multiple literacies - o "nuevas alfabetizaciones" — new literacies - (Coll y Rodríguez, 2008). Varis (2003) señala cinco «alfabetizaciones", cinco dimensiones o competencias requeridas para poder participar de forma activa, autónoma y eficiente en el vasto espectro de posibilidades que ofrecen las TIC:

- Alfabetización tecnológica: capacidad para utilizar nuevos medios para acceder a la información y utilizarla de forma eficaz.

- Alfabetización informacional: capacidad para recopilar, sistematizar y evaluar la información y generar a partir de ello opiniones válidas.

- Creatividad mediática: capacidad para elaborar y distribuir contenidos.

- Alfabetización global: comprender la interdependencia entre todos los actores, tecnologías y contenidos.

- Alfabetización responsable: capacidad para mesurar las consecuencias sociales de los medios.

Nuestro trabajo se enmarca en el segundo de los ámbitos descritos por Varis (2003) y, de manera específica, se centra en el aspecto relativo a la capacidad o competencia de recopilar o buscar información. Por otra parte, nuestro objetivo de estudio se ajusta a un escenario muy concreto, con unos actores determinados y un conjunto de necesidades singulares: la búsqueda de información con fines académicos — en bibliotecas e Internet- por parte del alumnado universitario pregraduado.

Existen un buen número de aproximaciones empíricas al campo de las estrategias de documentación académica por parte del alumnado universitario (Becker, 2003; Calva, 1999; Case, 2006; Chowdhury y Chowdhury, 1999; Cole, 1997; Dra- 
benstott, 2003; Kerins y otros, 2004; Liu y Yang, 2004; Valentine, 1993; Zhang y otros, 2005). De las que se centran en el análisis de la utilización de las bibliotecas como fuentes de documentación destacan las aportaciones de: Bancroft y otros (1998), quienes midieron el uso de la biblioteca de la Universidad de Washington por parte del alumnado de dicha institución; Hiller y Self (2002), que analizaron la frecuencia de utilización de las bibliotecas de las Universidades de Washington y Virginia entre los años 1998 y 2001; Hayden y colaboradores (2005), quienes describieron la frecuencia de uso por parte de los alumnos de los servicios bibliotecarios del Instituto Tecnológico de Waterford; Rey (1999), que describió el uso de las bibliotecas universitarias catalanas en el periodo comprendido entre 1995 y 1997; Perea (2002), que analizó el perfil del usuario del servicio de bibliotecas de la Universidad de A Coruña; Arias y Simón (2008), que estudiaron, mediante encuestación, los hábitos de uso de las bibliotecas de la Universidad Complutense de Madrid.

Por lo que respecta a la literatura sobre el uso de Internet como fuente de documentación académica en entornos de educación superior cabe distinguir, por una parte, los estudios centrados en el uso de la Red por parte de docentes e investigadores y, por otra parte, las investigaciones acerca del uso de Internet para la búsqueda de información entre alumnado universitario (Marzal y Calzada, 2003). Dentro de este segundo tipo de trabajos —en el que se incluye el que ahora presentamos - son destacables aportaciones como las de Dalgleish y Hall (2000), que mediante una aproximación cualitativa — basada en el método de entrevistas en profundidad- se adentraron en el análisis de la percepción del alumnado universitario acerca del empleo de Internet como fuente de documentación académica. En el mismo año, Wen-Hua (2000), también mediante enfoques cualitativos, analizó la autoeficacia en la competencia de búsqueda de información en contextos electrónicos. Posteriormente, Cothey (2002), mediante un estudio longitudinal, midió las estrategias documentales usando Internet puestas en práctica por 202 universitarios estadounidenses de primer curso. O'Hanlon (2002) midió a través de una prueba test las habilidades de los alumnos de la Universidad Estatal de Ohio para localizar información, mientras que Slaouti (2002), en Manchester, mediante un estudio de carácter cuantitativo, analizó la aceptación de Internet como fuente documental por parte del alumnado. Por lo que respecta a trabajos realizados en España, cabe destacar el de Martínez y otros (2002) que desarrollaron una investigación en la universidad de Oviedo acerca de los usos de las TIC por parte del alumnado de dicha institución, analizando, entre otras dimensiones, el uso de Internet como fuente documental. Marzal y Calzada (2003) realizaron un estudio entre alumnado de la Universidad Carlos III sobre necesidades y hábitos informativos de los universitarios cuando emplean la Red para documentarse. Sureda y Comas (2006) centraron su análisis en el alumnado de la carrera de Educación Social de la Universidad de las Islas Baleares y, por medio de un cuestionario, describieron el uso de Internet como fuente de documentación académica entre dicho colectivo. Finalmente, Comas (2009), en su tesis doctoral sobre el plagio académico entre el alumnado universitario, centró 
parte de su análisis en las fuentes de documentación — digitales e impresasconsultadas por los universitarios con fines académicos.

El propósito del presente trabajo consiste, en primer lugar, en conocer y precisar la autovaloración que hace el alumnado de sus competencias documentales con fines académicos cuando utiliza los servicios bibliotecarios e Internet y, en segundo término, precisar la frecuencia de uso de estos servicios así como de la Red para localizar información con finalidades académicas. Cabe reseñar que, tanto en el estudio realizado como en el presente artículo, se ha diferenciado en todo momento entre el uso de los servicios bibliotecarios e Internet, distinguiendo las bibliotecas universitarias - epígrafe bajo el que se incluye el acceso al fondo documental institucional físico y al fondo documental digital que puede consultarse de manera telemática y cuyo acceso depende de la institución académica- de las bibliotecas no universitarias de carácter general. Así, en el cuestionario utilizado para obtener los datos del alumnado, se apuntaba y especificaba claramente dicha diferenciación pera evitar posibles errores de interpretación. Así pues, el concepto de Biblioteca universitaria no se limita sólo a los recursos físicos que ésta pueda contener, sino que incluye todos aquellos recursos electrónicos accesibles desde su catálogo digital —ya sean propios o ajenos-, mientras que bajo el calificativo Internet se engloban todos aquellos portales y webs a los cuales el alumnado puede acceder sin hacer uso de recursos asociados a un servicio bibliotecario en particular.

\section{Método}

\subsection{Muestra y población}

En el estudio han tomado parte 1.025 alumnos pregraduados de la Universidad de las Islas Baleares (UIB) seleccionados mediante muestreo aleatorio a partir de la población compuesta por el total de los 11.380 matriculados en Grados, Diplomaturas, Licenciaturas e Ingenierías en la UIB. El tamaño de la muestra con el que se ha trabajado supone un error muestral de $\pm 2,9 \%$, para los datos totales, estimado para un nivel de confianza del $95 \%$ y bajo la condición más desfavorable de $p=q=0,05$.

El análisis de la frecuencia de uso de las bibliotecas, el catálogo de la biblioteca de la UIB e Internet para buscar información con fines académicos (véase punto 3.2. y subsiguientes) se ha realizado con una muestra de 730 alumnos. Esta disminución del número de encuestados se debe a que las preguntas del cuestionario se referían a la frecuencia de uso de los recursos a lo largo del curso anterior (2008-2009) siendo ya alumnado universitario y, por consiguiente, los alumnos que cursaban primero en el momento en el que se realizó el trabajo de campo (noviembre de 2009) no cumplían este requisito. La población total de alumnado matriculado en segundo, tercero, cuarto y quinto en la UIB es de 7.736 , lo que significa que la muestra con la que se ha trabajado - en 
esta sección concreta del trabajo- suponga un error muestral de $\pm 3,5 \%$ estimado para un nivel de confianza del $95 \%$ y bajo la condición más desfavorable de $p=q=0,05$.

La recogida de datos se realizó en el Campus de la UIB, durante el mes de noviembre de 2009, mediante la aplicación individual y anónima de un cuestionario por parte de 4 personas previamente instruidas. En la tabla I se exponen las características más destacadas de la muestra.

\subsection{Fuente de datos y variables de estudio}

Los datos para la elaboración del presente trabajo provienen de la "Encuesta sobre las competencias en Alfabetización Informacional del alumnado de la Universidad de las Islas Baleares", diseñada para el proyecto de investigación financiado por la Fundación IBIT en el marco del Plan Avanza del Ministerio de Industria, Turismo y Comercio. La principal finalidad de este proyecto consistía en analizar diversos aspectos sobre la alfabetización informacional del alumnado pregraduado de la UIB; para ello se establecieron cinco ámbitos de análisis que se ajustan a las cinco competencias básicas descritas y acordadas internacionalmente en el campo de la alfabetización informacional: a) Identificación de las necesidades de información; b) Estrategias de búsqueda de información; c) Evaluación y tratamiento de la información; $d$ ) Utilización y elaboración de la información, y e) Aspectos éticos y sociales de uso de la información (ACRL/ALA, 2000). El instrumento de recogida de datos constaba de un total de 41 preguntas entre las que había de tipo abiertas, cerradas dicotómicas, cerradas politómicas, de posición y escalas de respuesta.

Las variables seleccionadas para conformar este trabajo quedan enmarcadas en el campo competencial "Estrategias de búsqueda documental"; concretamente:

- Valoración por parte del alumnado de conocimientos y habilidades personales para buscar información en la biblioteca y en Internet (puntos $3.1 \mathrm{y}$ subsiguientes de este trabajo).

- Frecuencia de uso de: bibliotecas en general, el servicio de biblioteca de la UIB e Internet para buscar información con finalidades académicas por parte del alumnado (puntos 3.2 y subsiguientes de este trabajo).

Seguidamente, se relacionaron los resultados con diversas variables de posición y características descriptivas del alumnado participante: género, área de conocimiento y curso.

Cabe remarcar que, aún cuando el número total de alumnos encuestados asciende a 1.025, en algunas variables no se alcanza dicha cifra puesto que hubo sujetos que no respondieron a determinadas preguntas; es el caso, por ejemplo, de las variables categóricas "género" y "curso matriculado", donde se cuenta con 1.023 respuestas. 
TABLA I

Características y distribución de la muestra del estudio (tamaño de la muestra $=1.025$ alumnos)

\begin{tabular}{l|c|c}
\hline \multicolumn{1}{c|}{ Variable } & $\mathbf{N}^{\mathbf{0}}$ alumnos & $\mathbf{\%}$ \\
\hline Género: & & \\
Mujer & 670 & 65,5 \\
Hombre & 353 & 34,5 \\
\hline Área de conocimiento: & & \\
CC. Experimentales & 165 & 16,1 \\
CC. Sociales y Juridicas & 680 & 66,3 \\
CC. Salud & 134 & 13,1 \\
Humanidades & 46 & 4,5 \\
\hline Curso matriculado: & & \\
Primero & 295 & 28,8 \\
Segundo & 201 & 19,6 \\
Tercero & 257 & 25,1 \\
Cuarto & 218 & 21,4 \\
Quinto & 52 & 5,1 \\
\hline
\end{tabular}

\subsection{Análisis de datos}

Respecto a cada una de las variables de categoría se ha efectuado el cálculo de la frecuencia y el porcentaje, mientras que en las variables de escala se ha establecido el cálculo de la frecuencia, la media y la desviación estándar. Seguidamente, a efecto de poder establecer la posible asociación entre las variables en base a las que se operativiza la competencia de estrategias de búsqueda de información y las características del alumnado o variables categóricas (tabla II), se han diseñado tablas de contingencia para cada una de las variables y se ha efectuado la prueba de chi-cuadrado, calculándose el coeficiente de correlación de Pearson para, de esta forma, conocer el nivel de significación de las asociaciones generadas. Para la construcción de las tablas de contingencia se ha efectuado una subdivisión de la muestra en las variables analizadas; así, en la variable referente a la valoración de los conocimientos y habilidades para buscar información en las bibliotecas e Internet, se ha agrupado la muestra en "Valoración positiva" (casos en los que la valoración era "Buenos" y "Muy buenos"), "Valoración media" (casos en los que la valoración era "Aceptables") y "Valoración negativa" (casos en los que la valoración era "Nulos" y "Escasos"). En cuanto a la frecuencia de uso de las bibliotecas, de los servicios bibliotecarios de la UIB e Internet la agrupación hecha ha sido: "Usuario habitual" (casos en los que la frecuencia era "Entre 11 y 20 ocasiones", "Entre 21 y 50 ocasiones" y "Más de 50 ocasiones") y "Usuario no babitual" (casos en los que la frecuencia era "Nunca", "Menos de 5 ocasiones" y "Entre 5 y 10 ocasiones"). Para todos los análisis gene- 
rados en el presente estudio se ha empleado el paquete estadístico "Statistical Package for the Social Sciences" (SPSS versión 15.0).

\section{Resultados}

\subsection{Valoración por parte del alumnado de conocimientos y habilidades personales para buscar información con fines académicos en la biblioteca y en Internet}

La autovaloración que hacen los alumnos encuestados de sus conocimientos y habilidades para buscar información en Internet es bastante más positiva de la que hacen cuando se refieren al uso de las bibliotecas con el mismo fin (véase tabla II). Casi siete de cada diez alumnos (69,4\%) valoran sus habilidades y conocimientos a la hora buscar información en Internet como "Buenos" o "Muy buenos", mientras que referida al uso de las bibliotecas el porcentaje de estos rangos decrece hasta el 29,3\%.

Con el fin de identificar hipotéticas correlaciones —entre las autovaloraciones del alumnado con características como el género, curso y área de conocimientose han elaborado tablas de contingencia para cada una de ellas. En la tabla III se observa que existe una alta correlación entre la autovaloración de los alumnos y las variables género, el curso en que están matriculados y el área de conocimiento. Por lo que respecta a la asociación entre la autovaloración — "positiva", "media" y "negativa" - de las habilidades y conocimientos para buscar información con fines académicos en Internet y las características del alumnado se constata una relación significativa en las variables categóricas curso matriculado y área de conocimiento; mientras que no existe significación en relación a la categoría género.

\section{TABLA II}

Valoración por parte del alumnado de sus conocimientos y habilidades para buscar información con fines académicos en la biblioteca $y$ en Internet $(n=1.025)$

\begin{tabular}{l|c|c}
\hline \multicolumn{1}{c|}{$\begin{array}{c}\text { Valoración de conocimientos y habilidades } \\
\text { para buscar información }\end{array}$} & Bibliotecas \% & Internet \% \\
\hline Nulos & 4,7 & 0,4 \\
\hline Escasos & 22,5 & 3,1 \\
\hline Aceptables & 43,0 & 26,0 \\
\hline Buenos & 23,6 & 46,2 \\
\hline Muy buenos & 5,7 & 23,2 \\
\hline No sabe o No contesta & 0,5 & 1,1 \\
\hline Total & 100 & 100 \\
\hline
\end{tabular}




\section{TABLA III}

Valor de la prueba de chi-cuadrado de Pearson para la asociación entre la valoración de las babilidades y conocimientos para buscar información con fines académicos en las bibliotecas e Internet y las características del alumnado

\begin{tabular}{l|c|c|c|c|c|c|c|c}
\cline { 2 - 8 } \multicolumn{1}{c}{} & \multicolumn{4}{c}{ Bibliotecas } & \multicolumn{4}{c}{ Internet } \\
\hline $\begin{array}{c}\text { Características } \\
\text { de la muestra }\end{array}$ & $\chi^{\mathbf{2}}$ & $\boldsymbol{p}$ & $\mathbf{g . 1 .}$ & $\mathbf{n}$ & $\chi^{\mathbf{2}}$ & $\boldsymbol{p}$ & g.1. & $\mathbf{n}$ \\
\hline Género & $18,011^{*}$ & 0,000 & 2 & 1.018 & 1,687 & 0,430 & 2 & 1.013 \\
\hline Curso matriculado & $37,696^{*}$ & 0,000 & 8 & 1.018 & $67,326^{*}$ & 0,000 & 8 & 1.013 \\
\hline Área de conocimiento & $27,680^{*}$ & 0,000 & 6 & 1.020 & $22,202^{*}$ & 0,001 & 6 & 1.020 \\
\hline
\end{tabular}

$\chi^{2}=$ Chi-cuadrado de Pearson; $p=$ Significación; g.l. = Grados de libertad; * Significativa al nivel 0,01 (bilateral); $\mathrm{n}=\mathrm{N}^{\circ}$ alumnos de la muestra.

\subsubsection{Valoración de las habilidades y conocimientos para buscar información con fines académicos en bibliotecas y género}

En la figura 1 se puede constatar que las estudiantes presentan frecuencias superiores de respuesta en las valoraciones personales de sus habilidades y conocimientos para buscar información con fines académicos de tipo medio; mientras que entre ellos, aun cuando también domina el tipo de valoración media, presentan mayores frecuencias que ellas en las valoraciones negativas. En general, los alumnos universitarios muestran una peor autopercepción de sus habilidades y conocimientos para buscar información académica en las bibliotecas que las universitarias.

\subsubsection{Valoración de las habilidades y conocimientos para buscar información con fines académicos en bibliotecas y curso matriculado}

Como puede observarse en la figura 2, la autopercepción positiva respecto a las habilidades y conocimiento para buscar información en las bibliotecas aumenta curso a curso.

\subsubsection{Valoración de las habilidades y conocimientos para buscar información con fines académicos en Internet y curso matriculado}

El alumnado de primer curso es el que presenta frecuencias más moderadas a la hora de valorar sus habilidades y conocimientos para buscar en Internet. Los que tienen un mejor autoconcepto en este campo son los alumnos de tercero, sobre todo, y los de quinto (véase figura 3). 


\section{FIGURA 1}

Relación entre el género y la autovaloración de los conocimientos $y$ babilidades para buscar información en la biblioteca e Internet con fines académicos (\%)

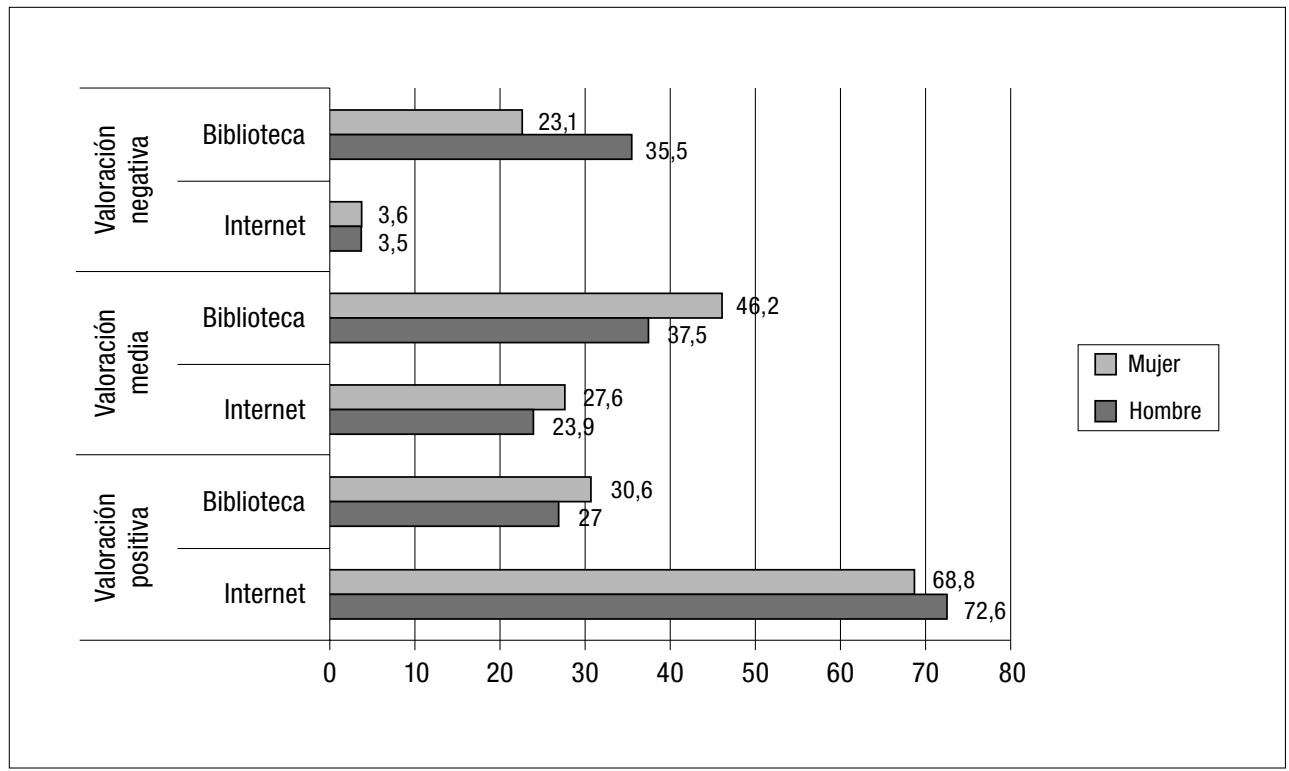

FIGURA 2

Relación entre el curso y la autovaloración de los conocimientos $y$ habilidades para buscar información en las bibliotecas (\%)

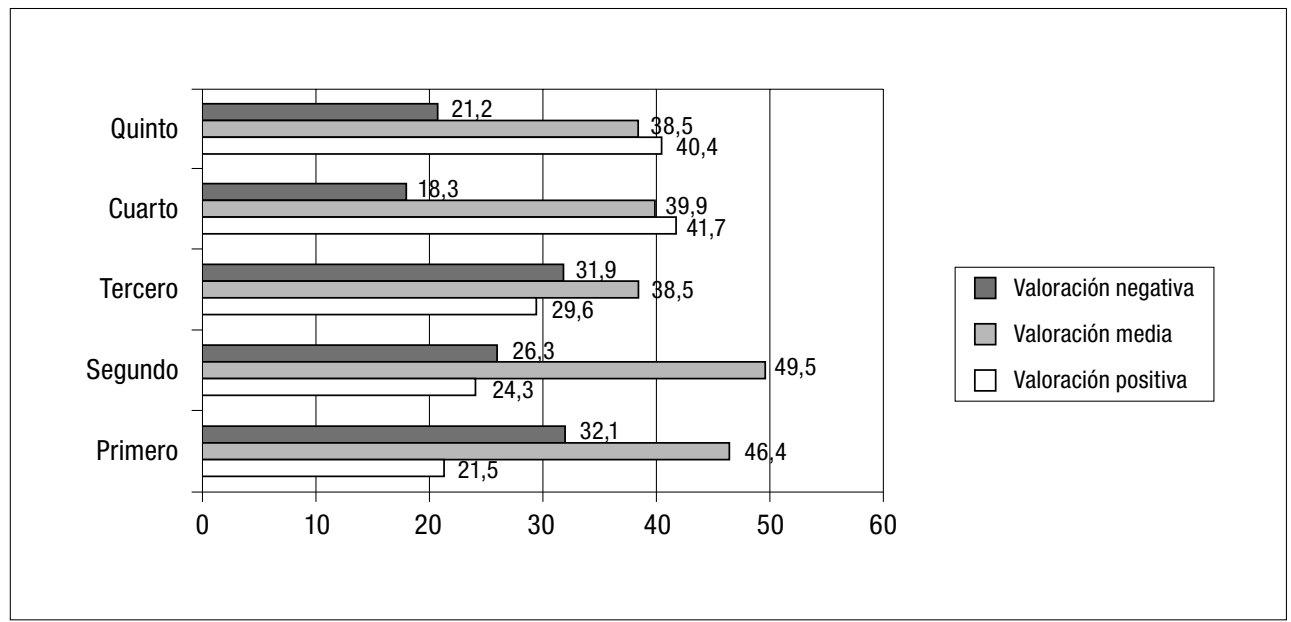




\section{FIGURA 3}

\section{Relación entre el curso y la autovaloración de los conocimientos} $y$ babilidades para buscar información en Internet (\%)

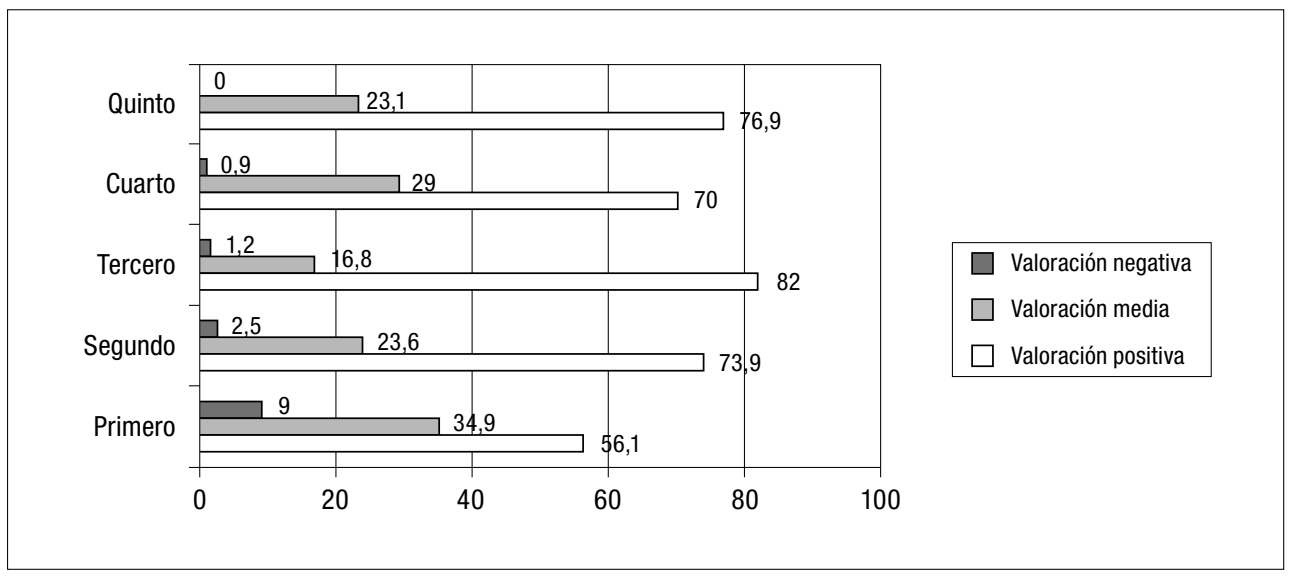

\subsubsection{Valoración de las habilidades y conocimientos para buscar información} con fines académicos en bibliotecas y área de conocimiento

El alumnado de Humanidades es, con una diferencia relevante respecto al resto, el que valora más positivamente sus conocimientos y habilidades en el uso de las bibliotecas para documentarse. En el polo opuesto se encuentran los alumnos de Ciencias Sociales y Jurídicas.

\section{FIGURA 4}

Relación entre el área de conocimiento y la autovaloración de los conocimientos y habilidades para buscar información en las bibliotecas (\%)

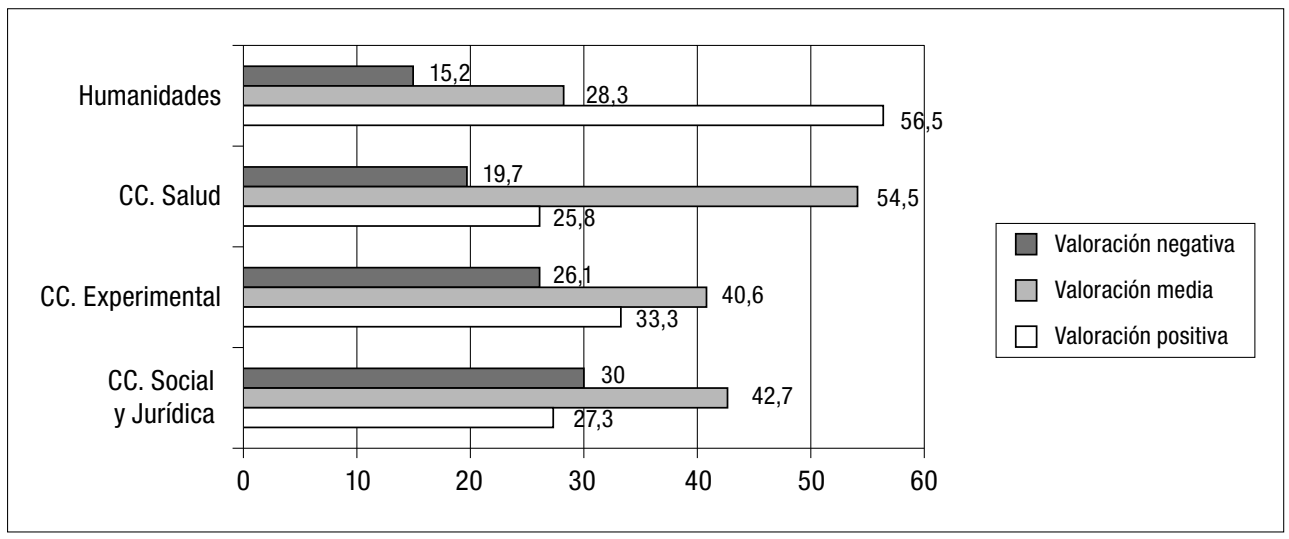


3.1.5. Valoración de las habilidades y conocimientos para buscar información con fines académicos en Internet y área de conocimiento

Los alumnos de carreras de las áreas de Ciencias Sociales y Jurídicas y Ciencias Experimentales son los que presentan frecuencias más altas en la valoración positiva de sus habilidades y conocimientos para buscar información académica en la Red.

\section{FIGURA 5}

Relación entre el área de conocimiento y la autovaloración de los conocimientos y habilidades para buscar información en Internet (\%)

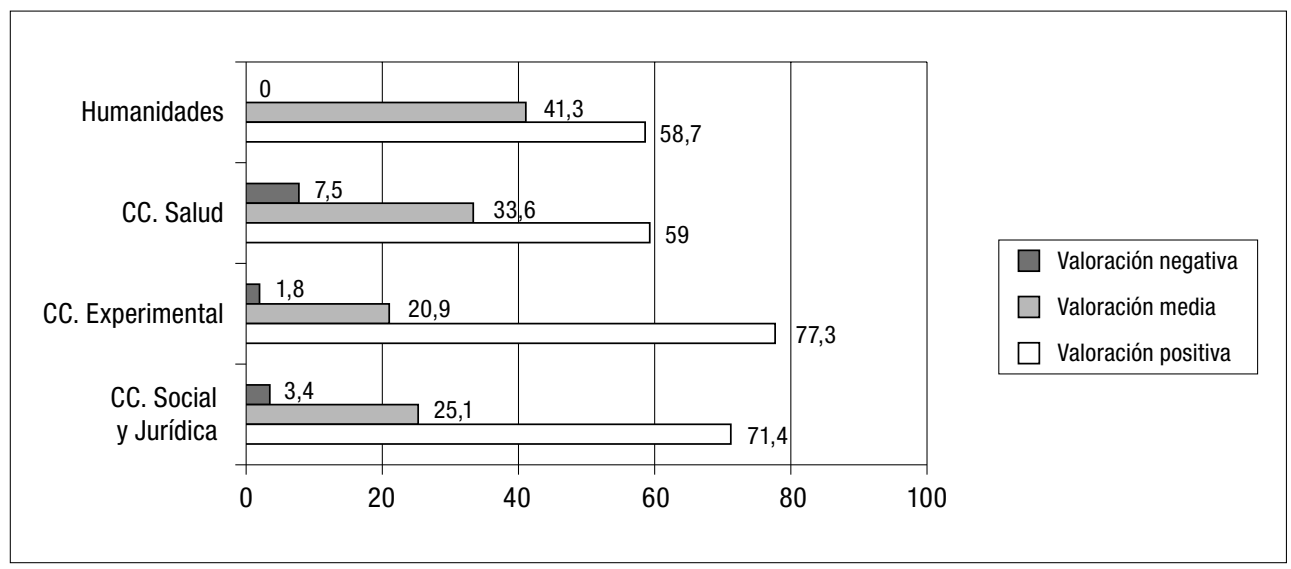

\subsection{Frecuencia de utilización de las bibliotecas, el catálogo del servicio de bibliotecas de la UIB e Internet para buscar información con fines académicos}

Los datos que se presentan en esta sección resultan de la formulación de tres cuestiones en las que se pedía la frecuencia de uso de las bibliotecas en general, el catálogo de la biblioteca de la UIB, e Internet, para buscar información con fines académicos a lo largo del curso 2008-2009. Por tanto, los datos hay que interpretarlos de manera diferida cuando se correlacionan con la variable curso. De esta manera, por ejemplo, el alumnado de segundo curso, cuando contesta, hace referencia a la frecuencia de uso que hizo de las fuentes de información nombradas a lo largo de primero de carrera; lo mismo sucede con los alumnos de tercero, cuarto y quinto.

La frecuencia con la que el alumnado de la UIB utiliza Internet a la hora de documentarse con fines académicos es apreciablemente superior a la del uso de las bibliotecas (véase tabla IV). Es destacable la escasa utilización del catálogo de la biblioteca de la propia universidad como fuente de documentación académica: casi 
cuatro de cada diez alumnos (37,9\%) nunca la usó durante el curso 2008-2009 y un 30,3\% lo hizo en menos de cinco ocasiones. En términos generales, se puede hablar de un uso extensivo de Internet como fuente de documentación a la hora de buscar información con fines académicos, muy superior al de las bibliotecas, en general, y al servicio bibliotecario de su propia universidad.

De cara a conocer una hipotética correlación entre la frecuencia de uso de las bibliotecas, el catálogo de la UIB e Internet para buscar información con finalidad académica y las características de los estudiantes, definidas en la tabla II, se han elaborado tablas de contingencia. En la tabla V se plasman los resultados y el valor de la prueba de chi-cuadrado de Pearson para cada una de las características descritas.

Por lo que respecta a la frecuencia de uso de las bibliotecas para buscar información académica, se da - como queda reflejado en la tabla V- una fuerte asociación entre los "usuarios habituales" y los "usuarios no habituales" de este tipo de espacios y las siguientes variables: curso en que están matriculados y área de conocimiento. Por el contrario, no se da una asociación significativa entre la frecuencia de uso de las bibliotecas y el género.

En relación a la asociación entre la frecuencia de utilización del catálogo de la biblioteca de la UIB —usuarios habituales" y "usuarios no babituales" - y las características del alumnado, se advierte una relación significativa en las variables categóricas curso matriculado y área de conocimiento; mientras que no existe significación en relación a la categoría género.

En tercer lugar, al analizar la asociación entre la frecuencia de uso de Internet como fuente de información con fines académicos y las categorías de agrupación del alumnado, se comprueba que ésta es fuerte en relación al curso en que el alumnado está matriculado y el área de conocimiento de la disciplina que estudia; mientras que no se da significación en la variable género.

\section{TABLA IV}

Frecuencia de utilización de las bibliotecas, catálogo de la biblioteca de la UIB e Internet para buscar información académica $(N=730)$

\begin{tabular}{l|c|c|c}
\hline \multicolumn{1}{c|}{ Frecuencia de utilización } & Bibliotecas \% & $\begin{array}{c}\text { Bibliotecas } \\
\text { de la UIB \% }\end{array}$ & Internet \% \\
\hline Nunca & 13,7 & 37,9 & 3,4 \\
\hline Menos de 5 ocasiones & 33,4 & 30,3 & 8,9 \\
\hline Entre 5 y 10 ocasiones & 23,0 & 17,0 & 16,0 \\
\hline Entre 11 y 20 ocasiones & 16,0 & 7,5 & 19,7 \\
\hline Entre 21 y 50 ocasiones & 7,9 & 4,7 & 20,3 \\
\hline Más de 50 ocasiones & 5,6 & 2,6 & 31,2 \\
\hline No sabe o No contesta & 0,4 & 0 & 0,5 \\
\hline Total & 100 & 100 & 100 \\
\hline
\end{tabular}




\section{TABLA V}

Valor de la prueba de chi-cuadrado de Pearson para la asociación entre la frecuencia de utilización de las bibliotecas, el servicio de bibliotecas de la UIB e Internet para buscar información con fines académicos y las características del alumnado

\begin{tabular}{l|c|c|c|c|c|c|c|c|c|c|c|c}
\cline { 2 - 13 } & \multicolumn{4}{c}{ Bibliotecas } & \multicolumn{3}{c}{ Biblioteca de la UIB } & \multicolumn{4}{c}{ Internet } \\
\hline $\begin{array}{l}\text { Caracteristicas } \\
\text { de la muestra }\end{array}$ & $\chi^{\mathbf{2}}$ & $\boldsymbol{p}$ & g.1. & $\mathbf{n}$ & $\chi^{\mathbf{2}}$ & $\boldsymbol{p}$ & $\mathbf{g . 1 .}$ & $\mathbf{n}$ & $\chi^{\mathbf{2}}$ & $\boldsymbol{p}$ & g.1. & $\mathbf{n}$ \\
\hline Género & 0,164 & 0,686 & 1 & 727 & 0,000 & 1,000 & 1 & 728 & 0,714 & 0,398 & 1 & 726 \\
\hline Curso matriculado & $35,125^{*}$ & 0,000 & 3 & 726 & $23,028^{*}$ & 0,000 & 3 & 727 & $21,062^{*}$ & 0,000 & 3 & 725 \\
\hline Área de conocimiento & $56,037^{*}$ & 0,000 & 3 & 728 & $69,974^{*}$ & 0,000 & 6 & 729 & $39,683^{*}$ & 0,000 & 3 & 727 \\
\hline
\end{tabular}

$\chi^{2}=$ Chi-cuadrado de Pearson; $p=$ Significación; g.l. = Grados de libertad; * Significativa al nivel 0,01 (bilateral); $\mathrm{n}=\mathrm{N}^{\circ}$ alumnos de la muestra.

\subsubsection{Frecuencia de utilización de las bibliotecas para buscar información con} fines académicos y curso

Como puede comprobarse en la figura 6, la frecuencia de uso de bibliotecas como fuente de documentación académica es más elevada en los cursos superiores (sobre todo en quinto) que en los cursos inferiores (fundamentalmente en segundo).

FIGURA 6

Relación entre el curso y la frecuencia de utilización de las bibliotecas para buscar información con fines académicos (\%)

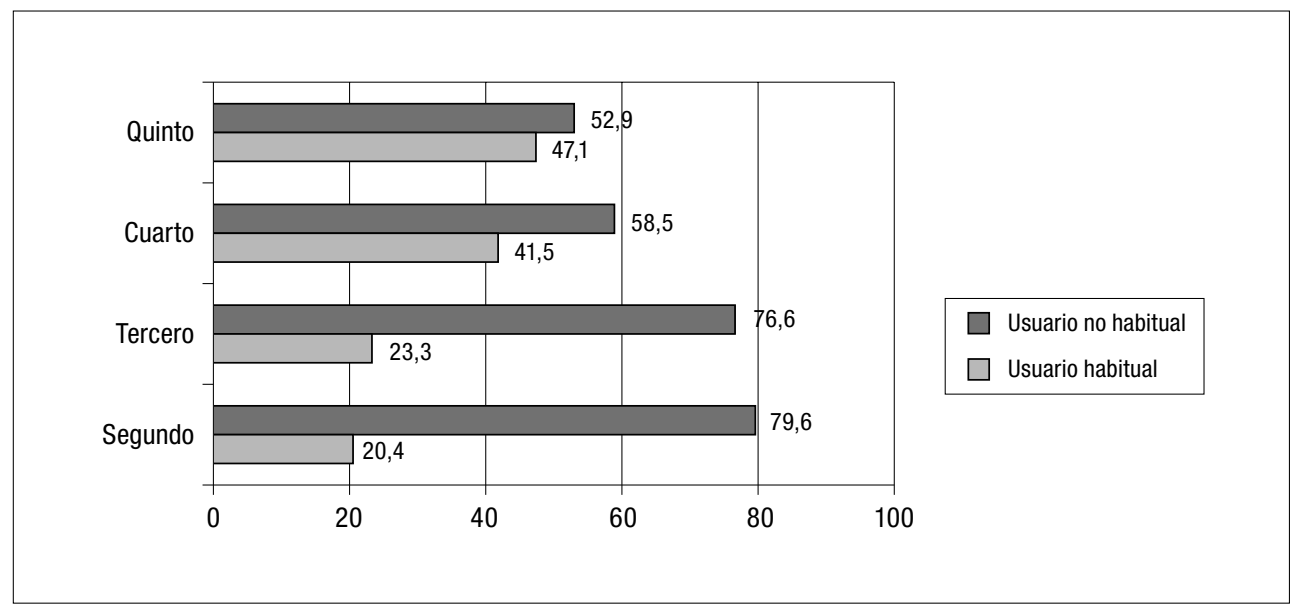




\subsubsection{Frecuencia de utilización del catálogo de la biblioteca de la UIB para buscar información con fines académicos y curso}

El alumnado de los cursos inferiores (segundo y tercero) es el que menos uso hace del catálogo de la biblioteca de la propia universidad a la hora de documentarse con fines académicos (véase figura 7). En este caso en concreto, el alumnado de cuarto curso es el que presenta una frecuencia más alta de utilización de la biblioteca de la Universidad de las Islas Baleares.

\section{FIGURA 7}

Relación entre el curso y la frecuencia de utilización de la biblioteca de la UIB para buscar información con fines académicos (\%)

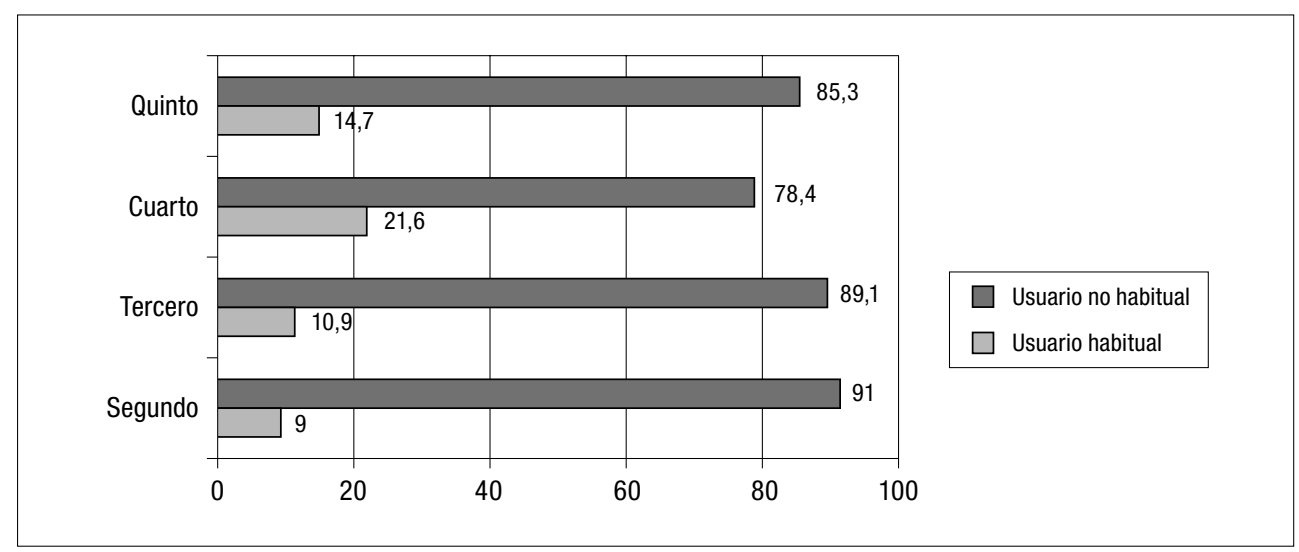

\subsubsection{Frecuencia de utilización de Internet para buscar información con fines académicos y curso}

El alumnado de quinto curso es el que muestra una mayor frecuencia en cuanto al empleo de Internet para localizar información con fines académicos; en segundo lugar, con frecuencias también bastante altas, se sitúan los alumnos de cuarto curso. Los estudiantes que manifiestan una menor frecuencia son los de tercero (véase figura 8).

\subsubsection{Frecuencia de utilización de bibliotecas para buscar información con fines académicos y área de conocimiento}

Los alumnos de Humanidades presentan frecuencias de uso de las bibliotecas para documentarse considerablemente superiores al resto de alumnado de otras áreas de conocimiento; prácticamente el 70\% de éstos son "usuarios babituales" de las bibliotecas. Los alumnos de Ciencias Sociales y Jurídicas son los que manifiestan emplear menos frecuentemente las bibliotecas para informarse: cerca del $80 \%$ afirma no utilizar habitualmente estos servicios (véase figura 9). 


\section{FIGURA 8}

Relación entre el curso y la frecuencia de utilización de Internet para buscar información con fines académicos (\%)

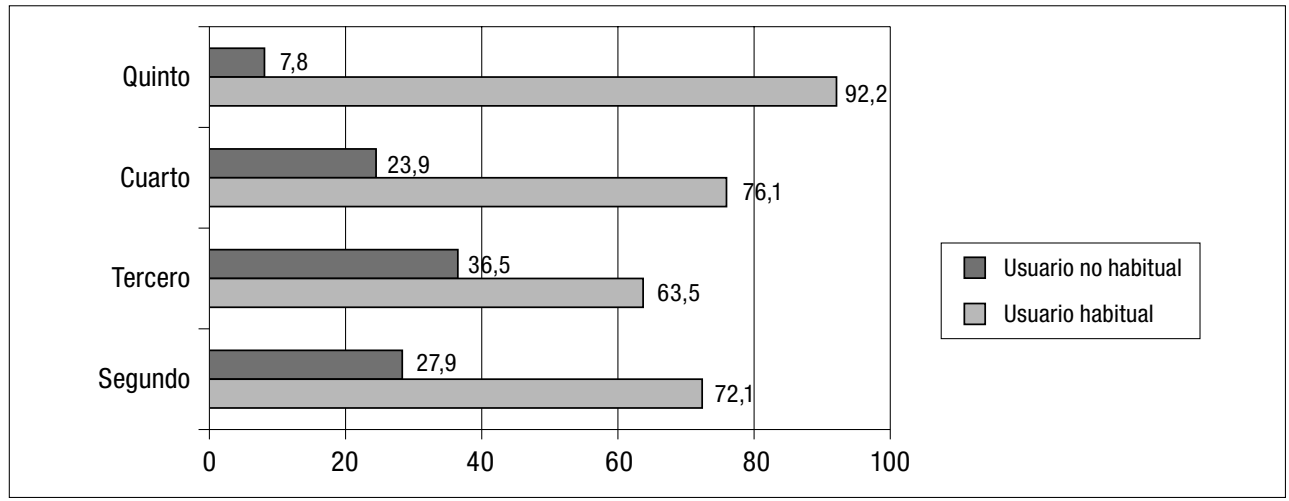

FIGURA 9

Relación entre el área de conocimiento y la frecuencia de utilización de bibliotecas para buscar información con fines académicos (\%)

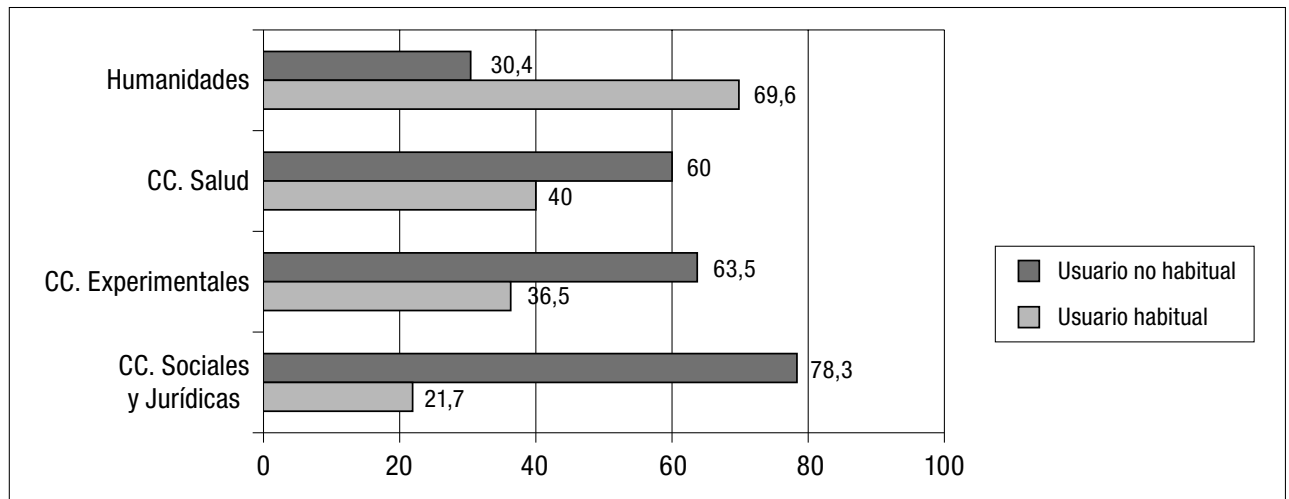

3.2.5. Frecuencia de utilización del catálogo del servicio de bibliotecas de la UIB para buscar información con fines académicos y área de conocimiento

En cuanto a la frecuencia de uso del catálogo de la biblioteca de la UIB sucede algo muy similar a lo comentado en el punto anterior: el alumnado de Humanidades es en su mayoría "usuario habitual" de este servicio, mientras que, como refleja la figura 10, el resto de alumnos, pertenecientes a disciplinas enmarcadas en otras áreas de conocimiento, muestran una frecuencia de utilización del recurso muy baja. 


\section{FIGURA 10}

Relación entre el área de conocimiento y la frecuencia de utilización del catálogo de la biblioteca de la UIB para buscar información con fines académicos (\%)

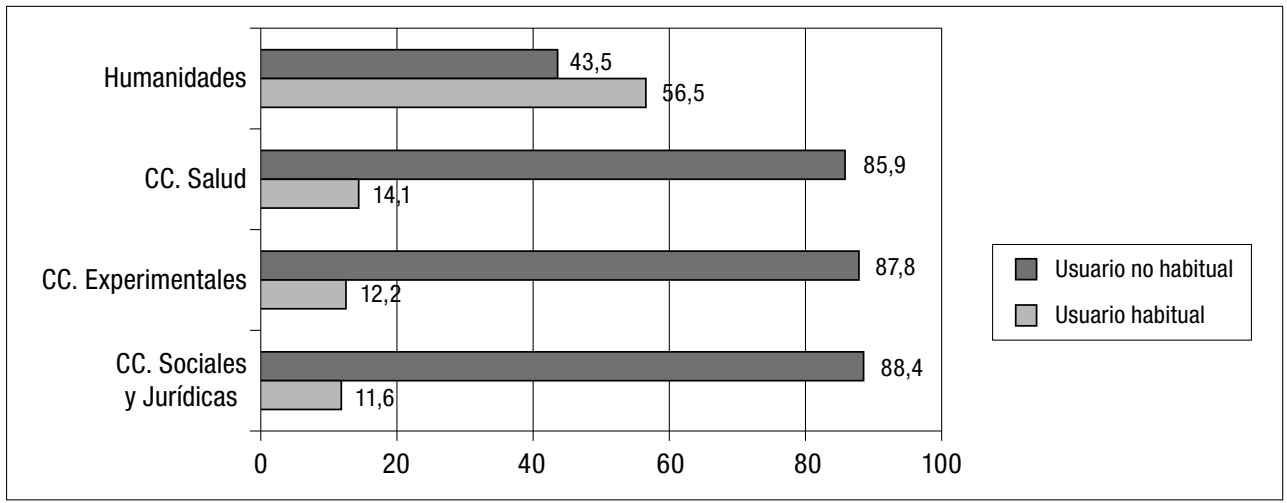

\subsubsection{Frecuencia de utilización de Internet para buscar información con fines académicos y área de conocimiento}

También en este caso, los alumnos de Humanidades lideran el ranking de frecuencia de utilización de Internet como fuente de información académica con más del 90\% de alumnos que son "usuarios habituales". Igualmente, son los alumnos de Ciencias Sociales y Jurídicas los que se sitúan en niveles de frecuencia más bajos (aun siendo muy superiores a los obtenidos en las variables relativas al uso de las bibliotecas y del catálogo de la biblioteca de la UIB) (véase figura 11).

\section{FIGURA 11}

Relación entre el área de conocimiento y la frecuencia de utilización de Internet para buscar información con fines académicos (\%)

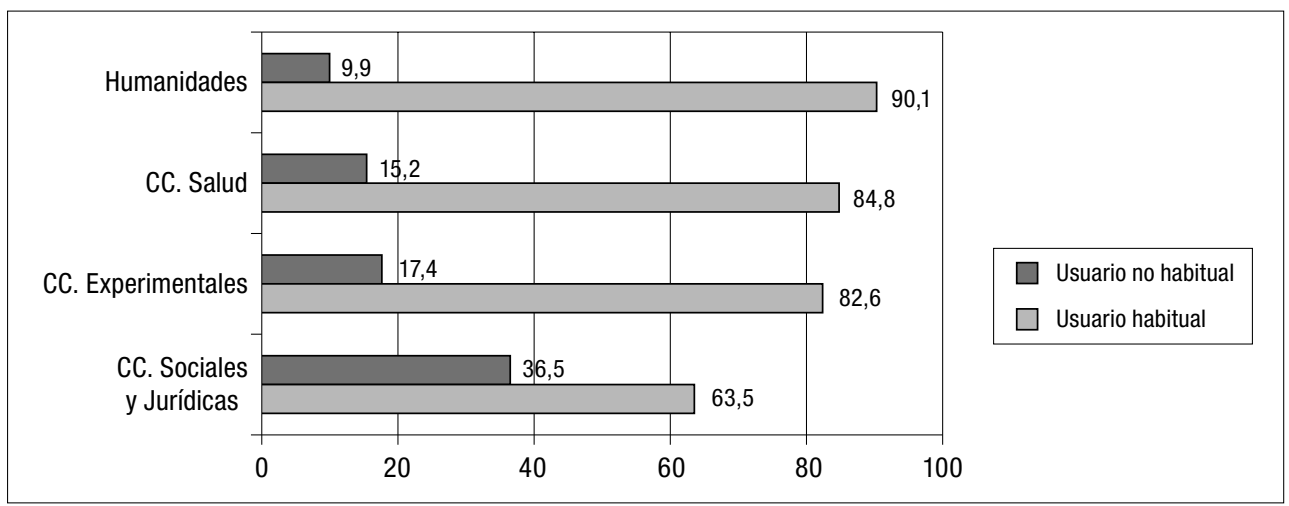




\section{Conclusiones y discusión}

Los resultados obtenidos reflejan que el alumnado universitario posee un mejor autoconcepto de su nivel competencial para usar Internet con fines académicos que para usar los servicios bibliotecarios. Este dato concuerda con los resultados de un estudio similar desarrollado en Alemania, aunque en ese caso los autores (Strube y Hoelscher, 2000) van un poco más allá al sugerir que los universitarios, por regla general, creen poseer mejores habilidades y conocimientos para encontrar información en Internet de los que realmente tienen. Datos similares se obtuvieron en el macroestudio — con una muestra de 27.317 alumnos de primer año de universidad- desarrollado por EDUCAUSE en los EEUU (Borreson y Salaway, 2008) donde más del 75\% del alumnado encuestado manifestó poseer habilidades "altas" y "muy altas" en el uso de Internet para documentarse. En la investigación de Sureda y Comas (2006), basada en un cuestionario contestado por alumnos matriculados en los estudios de Educación Social de la Universidad de las Islas Baleares, los datos obtenidos son muy parecidos: el 73,4\% afirmó poseer conocimientos y habilidades "buenas" y "muy buenas" a la hora de buscar en Internet información con fines académicos.

Cabe destacar las diferencias significativas que se dan entre la autopercepción competencial y determinadas características del alumnado. Se puede, en este sentido, trazar el siguiente perfil de alumnado con un autoconcepto positivo a la hora de usar las bibliotecas con finalidades académicas: mujeres y hombres que cursan los últimos cursos de carrera y que están matriculados en especialidades del área de conocimiento de Humanidades. En cuanto a los alumnos con un mejor autoconcepto de sus competencias a la hora de usar Internet con fines académicos cumplirían los siguientes patrones: hombres y mujeres matriculados en tercer curso que estudian carreras en las áreas de Ciencias Sociales y Jurídicas y Ciencias Experimentales.

Por lo que respecta a la frecuencia de utilización de las bibliotecas, los servicios bibliotecarios de la propia universidad e Internet para buscar información con fines académicos, resulta evidente, a primera vista, la preferencia del alumnado por el tercero de los recursos. Internet es, sin duda, la fuente predilecta entre el alumnado universitario pregraduado para buscar documentación académica. Este hecho concuerda con buena parte de los resultados de otros trabajos recientes sobre el tema como, por ejemplo, el ya mencionado estudio de Sureda y Comas (2006) con alumnos de la propia UIB; el análisis de las preferencias entre alumnado de secundaria británico a la hora de buscar información en medios electrónicos o las bibliotecas llevado a cabo por Shenton (2007); el trabajo de Saiti y Prokopiadou (2008) sobre las fuentes de información preferidas por alumnos post-graduados de universidades griegas; una investigación realizada en el Reino Unido con una muestra de 1.222 universitarios en la que Selwyn (2008) concluye que algo más del 50\% usa Internet para buscar información académica "Siempre" que lo necesita; la disertación doctoral de Comas defendida en 2009 (Comas, 2009); la investigación dirigida por Ismail centrada en alumnado de Tra- 
bajo Social de la Universidad de Marywood en los EEUU (Ismail, 2009).

El perfil de usuario frecuente de las bibliotecas y del catálogo bibliotecario de la propia universidad estaría compuesto por: hombres y mujeres matriculados en los últimos cursos de carreras de Humanidades. El estudio de Arias y Simón (2008) también presenta datos que hacen referencia a que el alumnado de Humanidades es el usuario más habitual de los servicios de bibliotecas de la Universidad Complutense de Madrid. Probablemente, el tipo de documentación que las disciplinas de Humanidades requieren - libros y manuales que sólo pueden encontrarse en las bibliotecas - y, también, el sentido y la metodología de los trabajos que se prescriben en estos estudios hacen que los alumnos de carreras de esta área sean los usuarios principales de los servicios bibliotecarios.

Cabe apuntar que, a la hora de valorar el uso de los servicios y recursos bibliotecarios frente al uso de Internet como fuente de documentación académica, debería tenerse en cuenta la dificultad de distinción, por parte del alumnado, entre lo que son recursos electrónicos proporcionados por las bibliotecas, y accesibles a través de Internet, de lo que son recursos de libre acceso en la Red. Es muy probable que esta distinción no sea claramente percibida por una parte considerable del alumnado que identifica la biblioteca con un espacio físico, o con el catálogo tradicional, pero no con otros recursos de biblioteca digital, recursos intensamente usados pero a los que se accede directamente como páginas marcadas en el navegador de Internet, o mediante accesos directos y no a través de los enlaces proporcionados desde el catálogo o la página de recursos del servicio de biblioteca. Este tipo de recursos, como el caso de "Westlaw" para las ciencias jurídicas o las grandes plataformas de revistas científicas "Web of Science", "Elsevier" o "Springer Link", para el caso de las ciencias experimentales, presentan un volumen muy alto de descargas de documentos que se incrementa sensiblemente año tras año y que no coinciden con los datos de uso de la biblioteca que manifiestan los alumnos en la encuesta.

También es conveniente fijarnos en otro factor que debería ponderarse: el escaso espacio físico con que cuentan las bibliotecas de la UIB en comparación con el resto de universidades españolas. Éste podría ser un factor que limitase el uso de los servicios bibliotecarios, no por la falta de interés en sus recursos documentales sino por las limitaciones de las infraestructuras para permitir un acceso y consulta de documentos.

Sea como fuere, en términos generales, como ya hemos señalado, a la hora de buscar información con finalidades académicas, el alumnado universitario se considera mucho más capaz de usar Internet que las bibliotecas; hace un uso extensivo de las TIC como fuente documental mientras que usa de forma casi marginal los servicios bibliotecarios. Internet constituye el universo informacional de los actuales alumnos universitarios, verdaderos "nativos digitales" (Prensky, 2001). Ahora bien, sería un error presuponer que, en este nuevo contexto, estos "nativos digitales" poseen una buena capacitación informacional. Es por ello que abogamos por la creación y consolidación de los Centros de Aprendizaje e Investigación (CRAI) y por la formación de base competencial del alum- 
nado. Creemos que es preciso proyectar iniciativas de este tipo para que las bibliotecas atraigan al alumnado y aseguren que sus competencias informacionales les permitan sacar el máximo provecho a su etapa de formación en la universidad.

\section{Bibliografía}

ACRL/ALA (2000). Information Literacy Competency Standards for Higher Education (en línea). Disponible en: http://www.ala.org/acrl/ilcomstan.html [consulta: 2/01/2010].

Arias, A., y Simón, J. (2008). Estudio de los hábitos de uso de los estudiantes en la biblioteca de la Universidad Complutense de Madrid. Revista Española de Documentación Científica, vol. 31 (3), 413-431.

Bancroft, A.; Croft, V. F.; Speth, R., y Phillips, D. M. (1998). A forward-looking library user Surrey: WSU libraries in the 21st Century. Journal of academic Librarianship, vol. 24, 216-224.

Becker, N. (2003). Google in perspective: understanding and enhancing student search skills. New review of Academic Librarianship, vol. 9 (1), 84-100.

Borreson, J., y Salaway, G. (2008). The ECAR study of undergraduate students and information technology. EDUCAUSE (en línea). Disponible en: http://www.educause.edu/ ers0808 [consulta: 12/01/2010].

Calva, J. (1999). El comportamiento y la búsqueda de información de los investigadores del área de humanidades y ciencias sociales. Investigación bibliotecológica, vol. 13 (27), 11-40.

Case, D. (2006). Information behavior. Annual Review of Information Science and Technology, vol. 40, 293-328.

Chowdhury, G., y Chowdhury, S. (1999). Digital library research: major issues and trends. Journal of Documentation, vol. 55 (4), 409-448.

Cole, C. (1997). Information as process: the difference between corroborating evidence and "information" in humanistic research domains. Information Processing and Management, vol. 33 (1), 55-67.

Coll, C., y Rodríguez, J. (2008). Alfabetización, nuevas alfabetizaciones y alfabetización digital: las TIC en el currículum escolar. En: Coll, C., y Monereo, C. (eds.). Psicología de la educación virtual, 325-347. Madrid: Morata.

Comas, R. (2009). El cibeplagio académico entre el alumnado universitario. Tesis Doctoral no publicada, Palma de Mallorca: Universidad de las Islas Baleares, Departamento de Pedagogía Aplicada y Psicología de la Educación.

Cothey, V. (2002). A longitudinal study of World Wide Web user's information searching behavior. Journal of the American Society for Information Science and Technology, vol. 53 (2), 67-78.

Dalgleish, A., y Hall, R. (2000). Uses and perceptions of the World Wide Web in an information seeking environment. Journal of Librarianship and Information Science, vol. 32 (3), 104-116.

Drabenstott, K. (2003). Do nondomain experts enlist the strategies of domain experts? Journal of the American Society for Information Science and Technology, vol. 54 (9), 836-854. 
Hayden, H.; O'Brien, T., y Rathaille, M. (2005). User Surrey at Waterford Institute of Technology Libraries. College Student Journal, vol. 24 (4), 557-570.

Hiller, S., y Self, J. (2002). A decade of user surveys: utilizing and accessing a standart assessment tool to measure library perfomance at the university of Virginia and university of Washington. Proceedings 4th Northumbria International Conference on Performance Measures in Libraries and Information Services. Washington DC, 12-16 de agosto de 2001.

Ismail, L. (2009). What are they telling us: Library use and needs of traditional and nontraditional students in a Graduate social work program. The Journal of Academic Librarianship, vol. 35 (6), 555-564.

Kerins G.; Madden, R., y Fulton, C. (2004). Information seeking and students studying for professional careers: the case of engineering and law students in Ireland. Information Research, vol. 10 (1). Disponible en: http://informationr.net/ir/10-1/paper208.html [consulta: 12/01/2010].

Liu, Z., y Yang, Z. (2004). Factors affecting distance-education graduate students' use of information sources: a user study. Journal of Academic Librarianship, vol. 30 (1), 24-35.

Martínez, R.; Pérez, H.; Granda, E., y Sampedro, A. (2002). El uso de las tecnologías de la información y la comunicación por parte de los estudiantes universitarios y su diferenciación en función del género (en línea). II Congreso europeo de tecnologías de la información en la educación y la ciudadanía: una visión crítica. Barcelona, 26-28 de junio. Disponible en: http://web.udg.edu/tiec/orals/c85.pdf [consulta: 12/01/2010].

Marzal, M., y Calzada, F. (2003). Un análisis de necesidades y hábitos informativos de estudiantes universitarios en Internet. Binaria: Revista de Comunicación, Cultura y Tecnologia, (3), 57-79.

O'Hanlon, N. (2002). Net knowledge: performance of new college students on an Internet skills proficiency test. Internet and Higher Education, vol. 5 (1), 55-66.

Perea, G. (2002). La biblioteca de la Universidade da Coruña. Estudio con los alumnos. Revista Española de Documentación Científica, vol. 25 (1), 29-42.

Prensky, M. (2001). Digital Natives, Digital Immigrants. On the Horizon, vol. 9 (5). Disponible en: www.marcprensky.com/.../Prensky\%20-\%20Digital\%20Natives,\%20Digital\%20Immigrants\%20-\%20Part1.pdf [consulta: 12/01/2010].

Rey, C. (1999). La aplicación de los estudios de satisfacción de usuarios en la biblioteca universitaria: el caso de las universidades catalanas. Biblioteconomia i Documentació, (3). Disponible en: http://www.ub.edu/bid/03rey2.htm [consulta: 12/01/2010].

Saiti, A., y Prokopiadou, G. (2008). Post-graduate students and learning environments: User's perceptions regarding the Choice of information sources. The International Information and Library Review, vol. 40, 94-103.

Selwyn, N. (2008). An investigation of differences in undergraduates' academic use of the Internet. Active learning in Higher Education, vol. 9 (1), 11-12.

Shenton, A. (2007). The information-seeking behaviour of teenagers in an English High School. School Librarian, vol. 5, 125-127.

Slaouti, D. (2002). The World Wide Web for academic purposes: old study skills for new? English for Specific Purposes, vol. 21 (2), 105-124.

Strube, G., y Hoelscher, C. (2000). Informationssuche und Wissenskommunikation: Wissenschaftlicher Alltag im Zeitalter der neuen Medien. En: Vob, G.; Holly, W., y Boehnke 
K. (editores). Neue Medien im Alltag: Begriffsbestimmungen eines interdisziplinären Forschungsprojekts, 177-198. Opladen: LeskeBudrich.

Sureda, J., y Comas, R. (2006). Internet como fuente de documentación académica entre estudiantes universitarios. Palma de Mallorca: Fundación Universidad Empresa de las Islas Baleares.

Valentine, B. (1993). Undergraduate research behaviour: using focus groups to generate theory. Journal of Academic Librarianship, vol. 19 (5), 300-304.

Varis, T. (2003). Nuevas formas de alfabetización y nuevas competencias en el e-learning (en línea). Disponible en: http://www.elearningeuropa.info/directory/index.php?page= doc\&doc_id=595\&doclng=7 [consulta: 4/01/2010].

Wen-Hua, R. (2000). Library instruction and college student self-efficacy in electronic information searching. The Journal of Academic Librarianship, vol. 26 (5), 323-328.

Zhang, X.; Anghelescu, H., y Yuan, X. (2005). Domain knowledge, search behaviour, and search effectiveness of engineering students: an exploratory study. Information Research, vol. 10 (2). Disponible en: http://informationr.net/ir/10-2/paper217.html [consulta $14 / 01 / 2010]$. 